the hospice with non-malignant conditions had risen from $11 \%$ (2015) to $21 \%$ (2018) during this time.

Staff training Teaching sessions were provided for staff to highlight key considerations when caring for patients with necrotising fasciitis, including symptoms and infection control advice. Feedback was gathered from staff attending teaching with $82 \%$ rating it as 'useful' or 'very useful' and $90 \%$ indicating that they would be interested in future education sessions.

Conclusion The breadth of patients referred for inpatient hospice management is growing. The case outlined may represent an emerging patient subgroup; those without a prior palliative diagnosis whereby active treatment of an acute complication has failed. Teaching sessions proved beneficial to hospice staff and should be considered in the future to promote individualised integration of care across disciplines.

\section{IMPROVING COMMUNICATION AND DOCUMENTATION OF END OF LIFE CONVERSATIONS, A SIMULATION BASED PROJECT}

E Haire, S Woods, N Wiggins. Great Western NHS Foundation Trust

10.1136/spcare-2020-PCC.45

Approximately $89.6 \%$ of patients' who are going to die in the next 12 months are admitted to hospital at least once. Evidence shows that good communication reduces hospital stays through advance care planning in the last 12 months of life. End of Life and DNAR discussions are part of the F2 curriculum and the development of good communication skills is a central part of clinical training. It is important to note that it is now a legal requirement that doctors involve patients and families in decisions regarding treatment escalation and resuscitation following the Tracey Judgement.

A recent audit undertaken at our Trust showed that there is scope for improving the quality of treatment escalation conversations the doctors are having with patients and their families as well as the documentation of these discussions.

An initial survey of F2 doctors showed that 92\% had previously had TEP discussions with a patient, $77 \%$ with a relative and $69 \%$ had completed a TEP form. 46\% reported not feeling confident having these discussions with main concerns not knowing what to say to initiate the conversation, being unable to answer questions and causing distress to both the patients and their family

A SIM course was delivered to improve the communication skills and confidence of F2 doctors allowing them to practice with actors in a safe learning environment. This learning was consolidated by debriefing with consultants in geriatric and palliative medicine. Post course feedback showed all participants enjoyed the course, $90 \%$ found it useful for clinical practice and $80 \%$ would recommend it to their colleagues. Significantly, all participants feel confident in discussing TEP following the SIM and commented on the detailed feedback.

This should lead to an increase in the number of patients who have these documented discussions which we know improves patient care.

\section{A MIXED-METHODS STUDY TO INVESTIGATE NURSING ATTITUDES TOWARDS ADMINISTRATION OF 'AS REQUIRED' PRESCRIBED SYMPTOM CONTROL MEDICATION AT END OF LIFE IN A HOSPITAL SETTING}

Elke Hall, Fiona Dakin, Christine Hirsch, John Speakman, Jon Tomas. University of Birmingham, University Hospital Birmingham NHS Trust

10.1136/spcare-2020-PCC.46

Background Anticipatory drugs for symptom control in dying patients are commonly prescribed in the acute hospital setting. The decision to administer 'as required' drugs usually lie with the ward nursing team who may have varied experience in end of life care. A previous survey informed changes to end of life care training offered within the Trust. This prompted the team to undertake a follow-up survey to determine whether the adapted training provision was meeting the needs of nursing staff administering 'as required' drugs at the end of life and to aid understanding of nurse decision making in this area of drug administration in the hospital setting.

Methods A questionnaire based on the previous survey was developed and piloted. A range of question styles were used including Likert scale, two ranking scales based on scenarios, and free text responses. Ethical approval was gained. Hard copies of the questionnaire were distributed to nurses on wards with the highest death rates together with elderly care wards. 100 copies of the questionnaire were distributed. Completed anonymous responses were coded and statistically analysed using SPSS 26.

Results The response rate was 62\%. Just under 50\% respondents reported being 'very confident' in recognising symptoms at the end of life. This was similar across the wards surveyed (oncology, acute medical, elderly care). Scenario ranking questions based on treatment of pain and agitation resulted in appropriate responses. 39\% of respondents had undertaken the Trust palliative care training. Five respondents (8\%) expressed fears around administration of medication at end of life.

Conclusion Most hospital nurses reported confidence in recognising end of life symptoms. Preliminary results have been shared with the specialist team, awaiting the final report to inform further development of training to improve the confidence and decision making of newly qualified nurses in this area of drug administration.

\section{PSYCHIATRY \& PALLIATIVE CARE: MEETING INTER-PROFESSIONAL, MULTI-DISCIPLINARY EDUCATIONAL NEEDS}

Daniel Hughes, Sarah Yardley, Katie Weatherstone. Central and North West London NHS Foundation Trust

\subsection{6/spcare-2020-PCC.47}

Introduction There is increasing evidence of higher rates of psychiatric disorder in palliative care patients. Navigating between palliative and psychiatric services can be challenging, and links between these specialities need to be strengthened to improve patient care and develop cross-disciplinary learning. Appropriate educational and networking opportunities for professionals to explore these issues has been limited. 
Methods Recognising an unmet educational need, a 'psychiatry in palliative care' study day was provided in 2018 (78 delegates) and 2019 (121 delegates), both were oversubscribed. All delegates were asked to complete an anonymous online questionnaire rating each topic, describing what was most and least useful; and how likely they would be to attend subsequent study days (completion rate $69 / 78$ in 2018, 84/121 in 2019).

Results Delegates were from a variety of healthcare backgrounds, disciplines and geographical regions with differing training levels. Across both years, reflecting learning needs, topics highly commended as extremely or very useful included 'personality disorder' (77/84, 91.6\%), 'treating delirium and dementia in palliative care' $(65 / 84,77.3 \%)$ and 'depression and psychosis in palliative care' (57/69 82.6\%). 142/153 $(92.8 \%)$ of participants across both days were extremely/very likely to attend subsequent events and/or recommend to a colleague. Qualitative feedback endorsed the value of interactive sessions and sharing learning about practical care and relationship building for service development. The event prompted development of a special interest module for psychiatry trainees embedded in palliative care teams, which is now in its second year, and sharing of audit tools to assess levels of combined palliative and psychiatric patient needs within palliative care services.

Conclusion These were important national learning events for best practice. We will continue, increasing capacity for 2020 . Our next study day will focus on latest advances in research, advice on setting up mental health support and risk assessment in the palliative care setting.

\section{TIME IS NOT AN EXCUSE, ADVANCE CARE PLANNING - THE USE OF A BRIEF INTERVENTION}

Catherine Hughes, Gill Horne, Wendi Abraham, Amanda Nadin, Kerry Macnish. Rowcroft Hospice, Torbay in partnership with Macmillan

\subsection{6/spcare-2020-PCC.48}

Background Lack of time is a recognised barrier to Advance Care Planning (ACP). ACP is a conversation that allows the person to specify preferences in relation to end of life care, stating wishes and choices.

Rowcroft Hospice with funding from Health Education England worked with people from different care settings to develop a brief intervention in ACP with the aim of openingup conversations with patients, giving staff prompts to help guide them.

Method Through a Macmillan funded project, we tested the Advance Care Planning Brief Intervention (ACP-BI) in practice for:

1. Usability by health care professionals (HCPs)

2. Acceptability by patients as reported by HCPs

3. To refine the training programme and BI, based on staff feedback

A steering group provided direction to the project. 24 HCPs were recruited from Nursing homes, Acute hospital, Community Hospitals and Community Nursing teams. A selfassessment tool was developed for the HCPs to test the intervention. Bespoke training was delivered and evaluated. Data on staff learning, including a reflective piece, were collected via a learning management system. Focus groups with HCPs were held midway and at the end of the project.

Results Not every person will engage with these conversations, HCPs included. However, the use of an ACP-BI, with training, and mentorship gives HCPs a building block to broach conversations about future planning, focusing on the patient's future and wishes. Qualitative feedback from nurses suggests it is possible to use a brief intervention for ACP: 'The initial conversation was only 15 minutes. I am surprised at how short these very important conversations can be'.

Conclusion Given that the median time of the 47 conversations was 12.5 minutes, time can no longer be used as a barrier to these conversations. We recommend the ACP-BI is further tested through research.

\section{HOW PAIN-RATING SCALES WORK (AND DO NOT WORK) IN REAL LIFE PRACTICE - A SMALL CONVERSATION ANALYTIC STUDY OF FIVE VIDEO- RECORDED HOSPICE CONSULTATIONS}

Laura Jenkins, Ruth Parry. Loughborough University

10.1136/spcare-2020-PCC.49

Background Assessing pain is a key palliative care task. Structured tools to assess pain intensity are commonly used, but patients report challenges in responding to these.

Aims The study aimed to analyse real-life episodes of pain scale use within hospice care, and develop communication training resources based on the findings.

Methods Pain scale use was identified in a dataset of videorecorded hospice consultations involving 37 patients and five doctors in a large UK hospice. The video data was subjected to Conversation Analysis - a direct observational approach to describing the challenges, structure and functioning of people's interactional behaviours.

Results Pain rating-scales were used in 5/37 consultations. We found that patients capitalise on scale use: taking it as an opportunity to communicate multiple aspects of pain - not just intensity. Video-clips exemplifying this are included within 'Real Talk' training materials (www.realtalktraining.co.uk), alongside learning points detailing how practitioners can support and encourage patients to do so. We found instances (2/ 5 ) of numerical scale misunderstandings. The experienced doctors we recorded handled these carefully. A video-clip demonstrating how a doctor and patient resolve a misunderstanding with caution and sensitivity is included in the Real Talk materials. Analysis-based learning points describe how practitioners can avoid resolving the misunderstanding in ways that imply the patient is to blame, and also consider how misunderstandings can take considerable time and interactional effort to resolve.

Conclusions Our analysis shows that pain rating-scales get used to report features besides their official target. Our findings directly underpin communication training resources for face-to-face training events - showing how professionals can support patients' maximal responses and how they can handle numerical rating misunderstandings in ways that avoid demeaning or disempowering patients. 